\title{
DIGESTIBILITÉ, VALEUR NUTRITIVE ET INGESTIBILITÉ DES BETTERAVES DE DIFFÉRENTES TENEURS EN MATIERE SECHE
}

\author{
C. DEMARQUILLY \\ avec la collaboration technique de J. M. Boissau, H. Bousquet et L. L'Hòtelier \\ Station de Recherches sur l'Élevage des Ruminants, \\ Centre de Recherches de Clermont-Ferrand., I. N. R. A., \\ 63 - Saint-Genès-Champanelle
}

\section{RÉSSUMÉ}

I. Nous avons mesuré la digestibilité et l'ingestibilité sur des moutons de 4 variétés de betteraves (Rouge d'Eckendorf, Monoval, Rod-Otofte et Monosvalof) représentatives de l'éventail des teneurs en matière sèche que l'on peut rencontrer actuellement. Les + variétés dont les caractéristiques sont présentées dans le tableau I ont été étudiées fraîches, 2 l'ont été aussi après déshydratation. Les betteraves ont été distribuées à volonté, les animaux recevant par ailleurs $200 \mathrm{~g}$ de foin. La digestibilité d'Eckendorf et Monosvalof ont en outre été mesurées sans distribution de foin aux animaux mais les betteraves étaient alors offertes en quantité limitée. L'emploi du temps de 3 des 6 moutons servant aux mesures a été enregistré graphiquement, et la composition du jus du rumen prélevé sur un septième mouton muni d'une fistule a été déterminée.

2. La teneur en matière sèche des betteraves fraîches a été très variable ( 55,5 à 28,4 p. Ioo) suivant les variétés. La teneur en glucides solubles $(59,4$ à 68,2$)$ a augmenté en même temps que la teneur en matière sèche. La déshydratation l'a fait diminuer. La teneur en azote a été relativement variable, I, 26 à $\mathrm{I}, 92$ p. Ioo suivant les variétés et indépendante de la teneur en matière sèche. Il en a été de même de la teneur en cellulose brute : 5,8 à 7,3 p. Ioo (tabl. I).

3. L'ingestibilité des betteraves fraîches distribuées à volonté a été très variable suivant les variétés (tabl. 2) : 75,0 à $4 \mathrm{I}, 4 \mathrm{~g}$ de matière sèche $/ \mathrm{kg} \mathrm{P}^{0,75}$ Elle a été $\mathrm{l}$ 'au tant plus élevée que les betteraves étaient plus pauvres en matière sèche et en sucres. Ces différences subsistent après déshydratation bien que celle-ci ait entrainé une diminution d'ingestibilité.

Le comportement alimentaire (tabl. 3) des moutons a été très différent de celui des moutons recevant des fourrages verts. La durée journalière d'ingestion a été très faible $(7,3$ à $10,8 \mathrm{p}$. I du temps total) et les betteraves ont été ingérées très rapidement notamment celles à faible teneur en matière sèche. Les repas ont été courts mais nombreux. La durée journalière de rumination, quoique faible, $(23, \mathrm{I}$ à 28,2 p. Ioo du temps total quand il y a eu un peu de foin dans la ration, I I , 3 à I 3,9 p. Ioo du temps total quand le foin a été supprimé) est restée cependant nettement supérieure à celle qui aurait été enregistrée si la même quantité de matière sèche avaient été offerte sous forme d'aliment concentré. Cela explique vraisemblablement que nous n'avons observé aucun trouble digestif chez les animaux au cours des 6 mois d'essai.

4. La digestibilité de la matière organique (tabl. 2) des betteraves fraîches distribuées sans foin a été de l'ordre de 90-91 p. roo. Celle des betteraves distribuées avec un peu de foin a été comprise entre 89,5 et 85,6 p. Ioo suivant les variétés par suite de phénomènes de digestibilité 
associative négative. La digestibilité de l'azote a été très variable (72,3 à 53,5 p. Ioo) mais n'a fait que traduire la variation de la teneur en azote. La digestibilité de la cellulose brute a été très élevée et peu variable, de l'ordre de $86 \mathrm{p}$. Ioo, quand les betteraves ont été distribuées sans foin.

La déshydratation a entraîné une diminution importante de la digestibilité de tous les constituants de la betterave. La diminution observée semble anormalement élevée et aurait vraisemblablement été réduite si les betteraves avaient été mises en cossettes plus petites et moins épaisses avant d'être déshydratées.

5. Le jus de rumen du mouton fistulisé recevant les betteraves a eu un pH très faible $(4,9$ à 5,5 ) et une composition en acides gras volatils caractérisée essenticlloment par une teneur élevée en acide butyrique et surtout en acides de longueur de chaîne égale ou supérieure à celle de l'acide valérianique et une teneur faible en acide acétique (tabl. 4).

\title{
INTRODUCTION
}

La betterave fourragère est intéressante à double titre; elle produit une quantité de matière sèche et d'unités fourragères à l'hectare qui est rarement atteinte par les autres productions fourragères et elle constitue un aliment énergétique très apprécié des ruminants.

La superficie qui lui est consacrée en France a fortement régressé jusqu'à ces dernières années (8I4 ooo ha en 1957,588 ooo en I967) car la culture de cette plante était très exigeante en main-d'œuvre. La betterave peut cependant intéresser à nouveau les exploitations pratiquant un élevage intensif par suite des possibilités toutes récentes de mécaniser entièrement sa culture (graines enrobées ou monogénétiques, désherbants spécifiques, semoirs de précision et arracheuses mécaniques). De nombreuses variétés de caractéristiques très différentes $(8$ à I 8 p. Ioo pour la teneur en matière sèche) sont actuellement commercialisées.

Peu d'auteurs ont mesuré la digestibilité des betteraves (cf. CoTTyn et Boucque, I970) et les mesures effectuées l'ont été sur des moutons recevant les betteraves en quantités très limitées. Ainsi, nous a-t-il paru intéressant de confirmer ces valeurs sur des moutons ingérant les betteraves à volonté, ce qui nous permettait d'étudier en même temps s'il existait des différences d'ingestibilité entre les variétés, notamment suivant leur teneur en matière sèche. Nous avons pour cela choisi 4 variétés représentatives de 1'éventail des teneurs en matière sèche que 1'on peut rencontrer actuellement ; elles ont, toutes quatre, été étudiées fraîches ; 2 l'ont été aussi après déshydratation.

\section{MATÉRIEL ET MÉTHODES}

\author{
Variétés de betteraves
}

Les caractéristiques des 4 variétés de betteraves étudiées : Ronge d'Eckendorf, Monoval, Rod-Otofte et Monosvalof sont présentées dans le tableau i.

Ces betteraves nous ont été fournies par la Maison de l'Élevage de l'Eure-et-Loir. Nous devions à l'origine recevoir ces 4 variétés sous formes fraîche et déshydratée. Les variétés ont pu être déshydratées après avoir été lavées au jet d'eau puis mises en fines cossettes avec un coupe- 


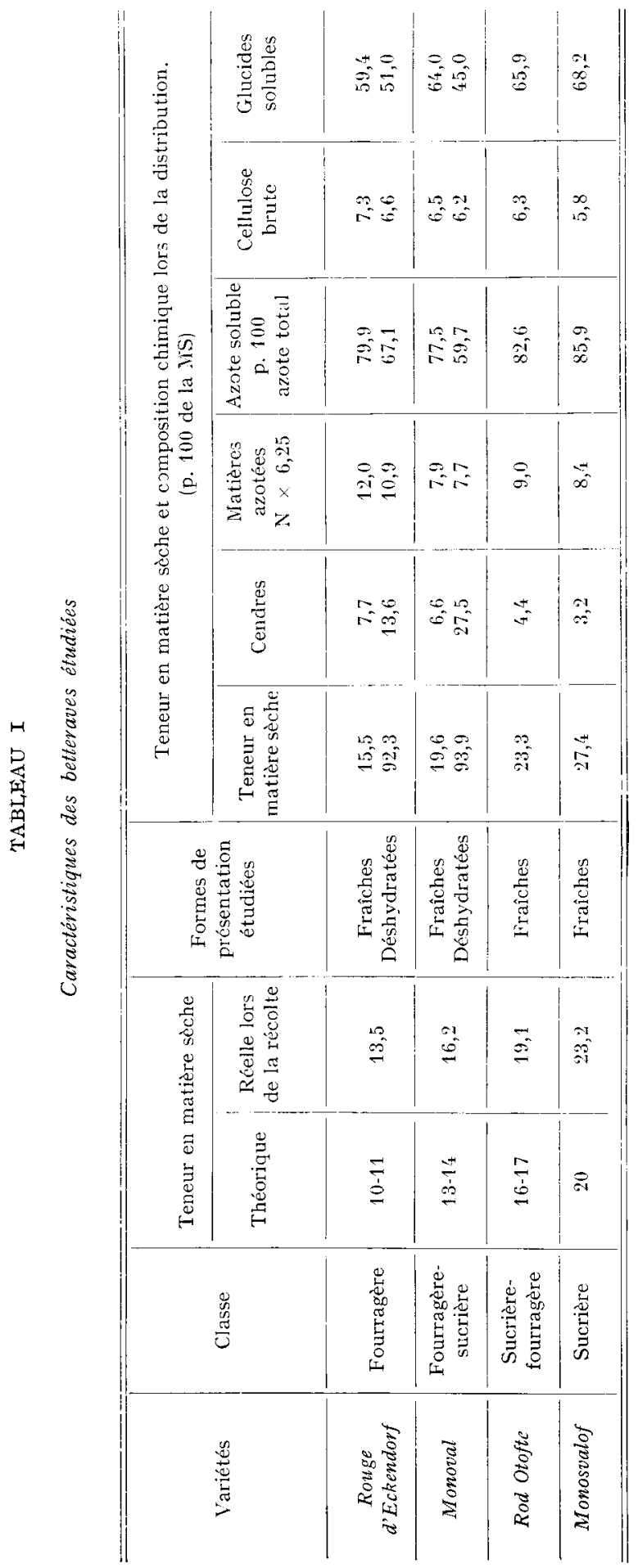


racines monté au pied du tablier élévateur de la déshydrateuse; cependant, les cossettes des variétés Rod-Otofte et Monosvalof (les plus riches en matière sèche et en sucre), quoique sortant très sèches de la déshydrateuse se sont ramollies en refroidissant, ce qui a entraîné une prise en masse rapide et une combustion complète du produit. Nous n'avons donc reçu sous forme déshydratée que les variétés Rouge d'Eckendorf et Monoval qui n'avaient d'ailleurs pas pu être granulées car les filières de la presse s'empâtaient rapidement.

Nous avons reçu les betteraves fin octobre. Les bətteraves déshydratées en cossettes ont été laissées en sac et les betteraves fraîches ont été storkées en tas dans un local fermé et légèrement chauffé (environ $10^{\circ} \mathrm{C}$ ) ; elles ont été distribuées aux animaux du $\mathrm{I}^{e r}$ décembre au 5 mars, suivant les échantillons, pour les betteraves fraîches, du 6 mars au Ier mai pour les betteraves déshydratées.

\section{Methodes de mesures}

La digestibilité et l'ingestibilité des 6 échantillons de betteraves ont été mesurées sur un lot de 6 moutons mâles castrés, de race Texel, âgés de 2 ans et demi et pesant environ $65 \mathrm{~kg}$. Les animaux ont reçu les betteraves à volonté (5 à Io p. Ioo de refus enlevés chaque matin) en 2 repas par jour, à 8 et à $\mathbf{I} 7$ heures. Les betteraves déshydratées ont été distribuées telles quelles, et les betteraves fraîches après avoir été soigneusement nettoyées et mises en fines cossettes avant chaque repas.

Craignant des indigestions, nous avons distribué à chaque mouton $200 \mathrm{~g}$ d'un foin de fléole mais ces $200 \mathrm{~g}$ n'ont pas toujours été entièrement consommés. La digestibilité du foin étant connue (respectivement $6 \mathrm{r}, 2,60,2$ et 67,4 pour les coefficients de digestibilité de la matière organique, des matières azotées et de la cellulose brute) nous avons calculé la digestibilité des betteraves en supposant qu'il n'y avait pas de digestibilité associative.

Nous avons en outre effectué pour 2 des 6 échantillons (Eckendorf et Monosvalof fraîches) une mesure de digestibilité sans distribution de foin aux animaux, mais les betteraves étaient alors offertes en quantités limitées de façon à être consommées en totalité. Dans tous les cas chaque période de mesures a duré 6 jours et était précédée d'une période préexpérimentale de I 5 jours.

L'emploi du temps de trois moutons, toujours les mèmes, a été enregistré graphiquement par la méthode de Ruckebusch (1963), pendant 5 jours consécutifs de chacune des périodes de mesure de la digestibilité.

Les échantillons représentatifs du foin et des botteraves offertes, des betteraves refusées et des fèces, correspondant à chaque période ont été analysés pour déterminer leurs teneurs en cendres, en matières azotées et en cellulose brute (Weende). Nous avons en outre déterminé la teneur en glucides solubles dans l'eau (à $40^{\circ} \mathrm{C}$ ) par la méthode de Somogyi (I952) et la teneur en azote soluble dans l'eau (à $40^{\circ} \mathrm{C}$ ) d'un échantillon représentatif de chaque catégorie de betteraves, l'analyse des betteraves ayant été effectuée après lyophilisation. Nous avons prélevé après lá distribution du repas du matin et durant 2 jours consécutifs de chaque période de mesure, dujus de rumen sur un $7^{\mathbf{c}}$ mouton muni d'une fistule du rumen. Le $\mathrm{pH}$ a été déterminé immédiatement et les échantillons conservés à - $15^{\circ} \mathrm{C}$ après addition de ro p. Ioo d'acide formique jusqu'au moment de la détermination en chromatographie en phase gazeuse de leur teneur en acides gras volatils par la méthode de RigAud et JOURNET (I970).

\section{RÉSULTATS ET DISCUSSION}

\section{Composition chimique de la matière sèche}

La composition chimique des betteraves est donnée dans le tableau $I$.

La teneut en matière sèche lors de la distribution aux animaux est très variable suivant les variétés $(\mathrm{I} 5,5$ à 28,4$)$ et est supérieure de 2 à 5 points à celle déterminée lors de la récolte par suite des pertes d'eau lors de la conservation. La teneur en cendres de betteraves fraîches qui avaient été soigneusement nettoyées et grattées à la main est faible : 3,2 à $8,0 \mathrm{p}$. Ioo suivant les variétés. Il n'en est pas de même pour les betteraves déshydratées; la variété Monoval contient en particulier 27,5 p. Ioo 
de cendres, ce qui prouve que le lavage au jet d'eau a été insuffisant pour débarrasser les betteraves de leur terre.

La teneur en glucides solubles $(59,4$ à 68,2$)$ augmente avec la teneur en matière sèche; pour une augmentation de I point de la teneur en matière sèche, la teneur en glucides solubles augmente significativement $(r=0,959$ pour $n=4)$ de 0,86 point en moyenne si on considère l'ensemble des 4 variétés et de 0,60 point ( $r=0,999$ pour $n=3$ ) si on excepte la variété Eckendorf typiquement fourragère. La déshydratation a entraîné une diminution de la teneur en glucides solubles vraisemblablement par suite des réactions de Maillard. D'après JARRIGE (résultats non publiés) 97 à 99 p. roo des glucides solubles seraient sous forme de saccharose dans les betteraves fraîches, de type sucrières ou sucrières-fourragères, contre seulement $6 \mathrm{I}$ à 62 p. Ioo dans les betteraves typiquement fourragères comme la Rouge d'Eckendorf.

La teneur en azote est relativement variable, I,26 à I,92 p. Ioo suivant les variétés, ce qui correspond à des teneurs en matières azotées $(\mathrm{N} \times 6,25)$ variant de 7,9 à I2,0 p. IOO. La variété Eckendorf est en particulier nettement plus riche en azote que les 3 autres. La teneur en azote est cependant indépendante de la teneur en matière sèche ou en sucres. $80 \mathrm{p}$. Ioo environ de l'azote se trouve sous forme soluble dans les betteraves fraîches contre 60 à $65 \mathrm{p}$. Ioo dans les betteraves déshydratées. D'après Fauconneau et Pion (résultats non publiés), le pourcentage de l'azote sous forme soluble dans les betteraves fraîches augmente avec la durée de la conservation; environ Io p. Ioo de cet azote sont constitués par des peptides, le reste par des acides aminés libres et surtout des amides (de la glutamine notamment).

La teneur en cellulose brute est faible et relativement peu variable : 5,8 à 7,3 p. Ioo elle diminue quand la teneur en matière sèche augmente. L'indéterminé représente I4,3 p. Ioo en moyenne $\left(I_{3}, 6\right.$ à I 5,0$)$ de la matière sèche.

\section{Ingestibilité des betteraves et comportement alimentaire des moutons}

Bien que ne recevant que $200 \mathrm{~g}$ de foin par jour (environ $\mathrm{I} 8 \mathrm{o} \mathrm{g}$ de matière sèche), les moutons n'en ont consommé que de 54 à $90 \mathrm{p}$. Ioo suivant les périodes, soit une quantité représentant de 6,5 à 13,7 p. Ioo (9,7 p. Ioo en moyenne) de la quantité totale de matière sèche ingérée. Pour une période donnée la quantité de foin ingérée a en outre été très variable d'un mouton à l'autre, puisqu'elle a varié de o à $200 \mathrm{~g} / \mathrm{jour}$. En revanche, la quantité de betterave ingérée a été très peu variable d'un mouton à l'autre, le coefficient de variation entre moutons n'a été que de $3,3 \mathrm{p}$. Ioo en moyenne, ce qui permet de supposer que les variations de la quantité de foin ingérée n'ont pas eu d'influence sur la quantité de betteraves ingérée.

L'ingestibilité des betteraves fraîches distribuées à volonté a été très variable suivant les variétés (tabl. 2) : la variété Eckendorf a été ingérée en quantité $(75,0 \mathrm{~g}$ de matière sèche $\left./ \mathrm{kg} \mathrm{P}^{0,75}\right)$ significativement $(\mathrm{P}<0,05)$ plus importante que les variétés Monoval et Rod Otofte (respectivement 54.3 et $59,3 \mathrm{~g}$ ), qui ont été elles-mêmes ingérées en quantitésignificativement plus importante que la variété Monosvalof $(4 \mathrm{I}, 4 \mathrm{~g})$. La quantité de matière sèche de betteraves fraîches ingérée a donc été d'autant plus importante que la teneur en matière sèche et la teneur en sucres de la variété étaient plus faibles. Ces différences d'ingestibilité entre variétés seraient intéressantes à confirmer, notamment sur des bovins, si on désire une utilisation maximum de la 


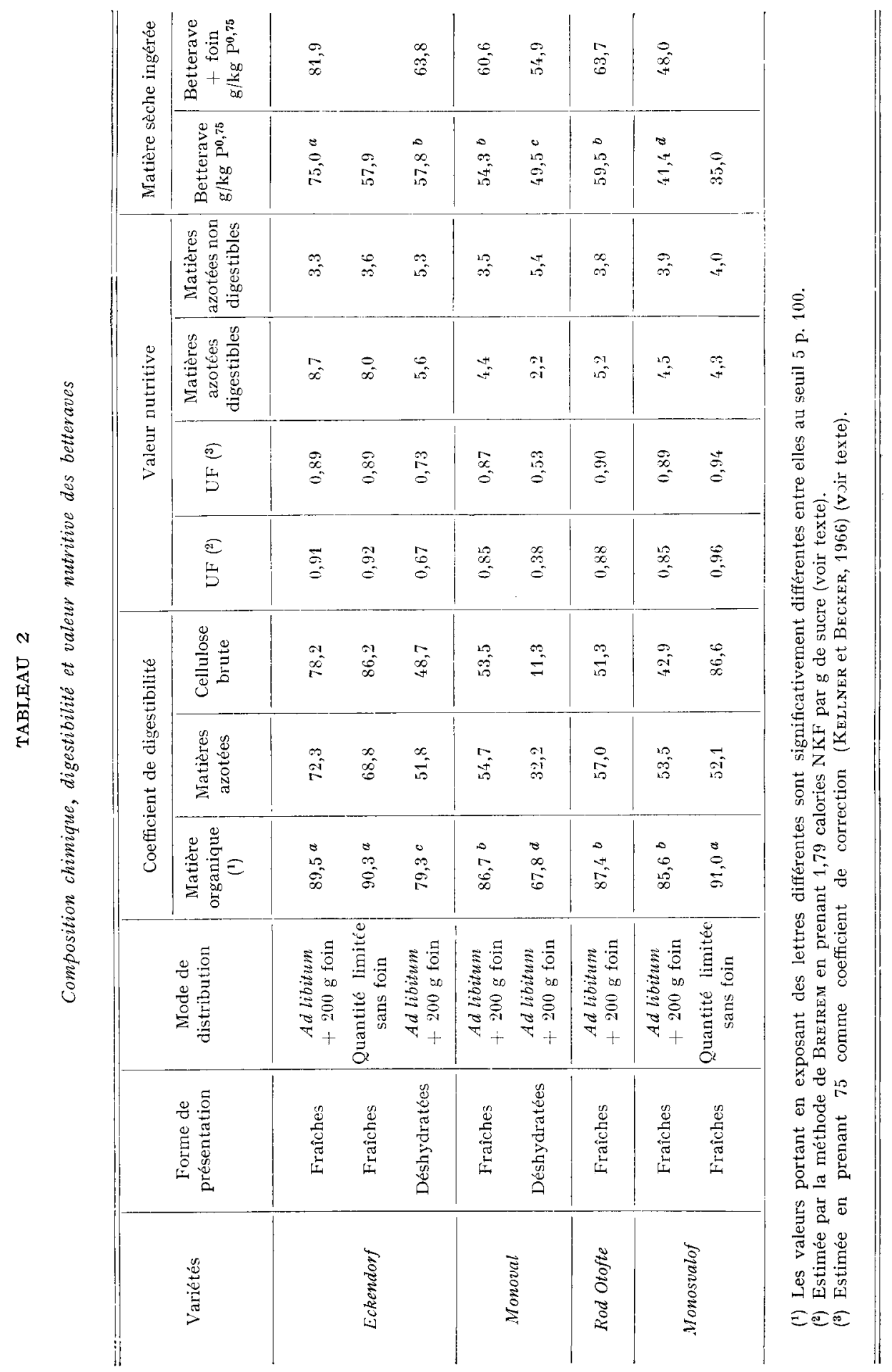


bettèrave dans la ration. Il est difficile de préciser la ou les causes de ces différences d'ingestibilité. Elles peuvent en effet se situer à 3 niveaux :

ro au niveau buccal et être alors d'ordre gustatif ; la dureté n'a vraisemblablement pas pu jouer à ce niveau puisque les betteraves ont été distribuées en fines cossettes; la vitesse d'ingestion a d'ailleurs été très élevée pour toutes les variétés (cf. plus loin);

$2^{\circ}$ au niveau digestif par suite de différences éventuelles dans les vitesses de digestion et de fermentation dans le rumen;

$3^{\circ}$ au niveau métabolique.

L es différences entre variétés subsistent après déshydratation bien que celle-ci ait entraîné une diminution d'ingestibilité variable : de 23 p. Ioo pour la variété Eckendorf et de 9. p. Ioo seulement pour la variété Monoval.

Le comportement alimentaire (tabl. 3) des moutons recevant des betteraves diffère sensiblement de celui des moutons recevant des fourrages verts même ceux de début de printemps qui ont une digestibilité et une ingestibilité élevées.

Quand les betteraves fraîches ont été offertes à volonté avec un peu de foin, la durée journalière d'ingestion a été très faible et n'a représenté que de 7,3 à Io,8 p. Ioo du temps total alors qu'elle descend rarement en-dessous de $20-22$ p. Ioo avec les fourrages verts. Les betteraves ont donc été ingérées très rapidement; la durée unitaire d'ingestion est en moyenne de $2, \mathrm{I} 6$ ( $\mathrm{I}, 73$ à 2,58) minutes par $\mathrm{g}$ de matière sèche ingérée par $\mathrm{kg} \mathrm{P}^{0,75}$ alors qu'elle est pratiquement toujours supérieure à 4,0 minutes pour les fourrages. Les betteraves à faible teneur en matière sèche ont été ingérées plus rapidement surtout si on considère la quantité de matière fraîche ingérée; c'est ainsi que pour ingérer I $\mathrm{kg}$ de matière fraîche de betteraves, les moutons ont mis respectivement I I,7, I7,3, 26,2 et 27,6 minutes pour des betteraves ayant des teneurs en matière sèche de $15,5,19,6,23,3$ et 27,4 p. Ioo. Par ailleurs, les repas ont été très courts, $\mathrm{I} 8$ minutes en moyenne ( 15 à 22 minutes selon les échantillons) et d'autant plus nombreux que l'ingestibilité des betteraves était élevée. La quantité moyenne de matière sèche ingérée par repas a donc été faible et relativement peu variable; de $7, \mathbf{I}$ à $8,2 \mathrm{~g} / \mathrm{kg} \mathrm{P}^{\mathbf{0}, 75}$ suivant les variétés. La déshydratation des betteraves, de même que la présence ou non d'une petite quantité de foin dans la ration, a eu peu d'influence sur les paramètres de l'ingestion. Seule la durée unitaire d'ingestion a été un peu augmentée, ce qui signifie que la vitesse d'ingestion est un peu plus faible quand les betteraves sont déshydratées ou quand le foin est supprimé de la ration.

La durée journalière de rumination, a, elle aussi, été faible; elle n'a représenté que $23, \mathrm{I}$ à 28,2 p. roo du temps total quand il y a eu un peu de foin dans la ration et elle est tombée entre I I,3 et I3,9 p. Ioo du temps total quand le foin a été supprimé. Il convient de souligner que la distribution exclusive de betteraves ne supprime pas entièrement la rumination comme le ferait un régime uniquement à base d'aliments concentrés. Le temps passé à ruminer reste du même ordre que celui observé avec les régimes comprenant $80 \mathrm{p}$. Ioo d'aliments concentrés et 20 p. Ioo de foin ou de paille (BINES et DAVEX, I970; - RÉMOND, résultats non publiés). La distribution d'un peu de foin (Io p. Ioo environ de la matière sèche de la ration) en supplément des betteraves permet en outre de doubler le temps passé à ruminer qui atteint alors une valeur très acceptable (environ $25 \mathrm{p}$. Ioo du temps total). La durée unitaire de rumination a, de même, été faible; elle est restée comprise entre 3,5 et 9,o minutes par g de matière 


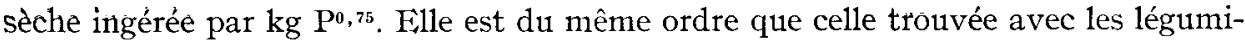
neuses en début de printemps et doit traduire une vitesse de digestion très rapide dans le rumen.

Quand les betteraves, qu'elles aient été fraîches ou déshydratées, ont été distribuées à volonté et donc avec un peu de foin, il y a eu compensation entre la durée journalière d'ingestion et la durée journalière de rumination. La durée journalière de mastication (ingestion + rumination) a donc été le paramètre le moins variable du comportement alimentaire ; elle est restée comprise entre 32,0 et 37,4 p. Ioo du temps total et a été indépendante de la quantité ingérée. Il en résulte que la durée unitaire de mastication (DUM) a été très étroitement liée à la quantité ingérée $(r=-0,988$ pour $n=6)$.

$$
\text { DUM en minutes }=\mathrm{I} 7,86-0, \mathrm{I} 50 x
$$

où $x=$ quantité de matière sèche ingérée en $\mathrm{g} / \mathrm{kg} \mathrm{P}^{3,75}$.

\section{Digestibilité ct valeur nutritive}

Les coefficients de digestibilité des betteraves sont donnés dans le tableau 2. L'analyse de la variance des résultats n'a pu être faite que pour le coefficient de digestibilité de la matière organique car nous n'avons pas déterminé les teneurs en azote et en cellulose brute des fèces de chaque mouton mais celles de l'échantillon représentatif des fèces des 4 moutons en cage. Les différences de digestibilité entre moutons ont été extrêmement faibles : l'écart-type d'une mesure est de 0,7 point ce qui correspond à un coefficient de variation de 0,83 p. Ioo seulement.

I a digestibilité de la matière organique des 4 variétés de betteraves fraîches distribuées avec une petite quantité de foin est élevée, la valeur de la variété Eckendorf $(89,5)$ étant significativement plus élevée $(\mathrm{P}<0,05)$ que celle des 3 autres : 86,7, 87,4 et 85,6 . La suppression du foin n'a pas augmenté significativement la digestibilité de la variété Eckendorf ( $+0,8$ point) mais significativement $(\mathrm{P}<0,05)$ la digestibilité de la variété Monosvalof ( $+5,4$ points).

Il y a donc eu un phénomène de digestibilité associative négative entre la betterave et le foin mais d'importance très variable suivant la variété considérée et qui est d'ailleurs confirmée et expliquée en partie par les différences entre les coefficients de digestibilité de la cellulose brute obtenus pour ces 2 variétés, quand elles ont été distribuées avec ou sans foin : respectivement 78,2 contre 86,2 p. Io0 pour la variété Eckendorf, 42,9 contre 86,6 p. Ioo pour la variété Monosualof. N'ayant distribué que 2 variétés sans foin il est difficile de connaitre les causes exactes de ces différences de digestibilité associative. Celles-ci ne peuvent venir de différences dans le niveau d'ingestion puisque la quantité de betteraves ingérée en l'absence de foin représente dans les 2 cas environ 80 p. Ioo de celle ingérée en présence de foin. Elles peuvent donc résulter soit du fait que le foin a représenté, suivant les variétés, une proportions différente de la matière sèche totale de la ration : 8,4 p. Ioo pour Eckendorf contre I3,7 p. Ioo pour Monosvalof, soit du fait que la variété Monosvalof était plus riche en sucre ou plus pauvre en azote. On sait en effet que la digestibilité des membranes d'un foin diminue en présence de glucides très fermentescibles et cela surtout si la teneur en azote de la ration est faible. Quoi qu'il en soit, il est vraisemblable que la digestibilité de la matière organique de la betterave distribuée seule est peu variable, de l'ordre 
de 9o-gr p. Ioo quelle que soit la variété (tabl. 2). Ces valeurs sont d'ailleurs très voisines de celles mesurées par DijKSTRA (r953 et I960), FREDERIKSEN et al. (I964), KEII,NER et BECKER (I966) et par COTTYN et BouCque (I97o) ; respectivement 89,9, 90,3 et $86,7,92,0,90,0,92,4$. De même, il semble que la digestibilité de la cellulose brute de la betterave est très élevée et peu variable, de l'ordre de 86,0 p. Ioo, les valeurs plus basses et plus variables $(78,2$ à 42,9) observées quand les mesures ont été faites en distribuant une petite quantité de foin, ne traduisant elles aussi que des phénomènes de digestibilité associative.

La digestibilité de l'azote a été très variable (72,3 à 53,5 p. Ioo) (tabl. 2) mais elle n'a fait que traduire la variation de la teneur en azote. La teneur en matières azotées digestibles (MAD en p. IOo) pourra être obtenue en soustrayant 3,7 de la teneur en matières azotées totales (MAT en p. IOo)

$$
\mathrm{MAD}=\mathrm{MAT}-3,7
$$

80 p. Ioo de l'azote étant sous forme soluble, facilement fermentescible, la teneur en matières azotées digestibles obtenue doit cependant être considérée comme une estimation généralement par excès, du moins quand les betteraves représentent une part importante de la ration.

La déshydratation a entraîné une diminution importante de la digestibilité de tous les constituants de la betterave et cela d'autant plus que la betterave était plus riche en sucre: diminution de la digestibilité de la matière organique de 10,2 points pour la variété Eckendorf et de I 8,9 points pour la variété Monoval. Quant à la teneur en matières azotées non digestibles, elle est passée de 3,40 à 5,35 p. Ioo en moyenne, soit une augmentation de $57 \mathrm{p}$. Ioo. S'il est normal que la déshydratation entraîne une diminution de la digestibilité plus importante pour la betterave, produit riche en eau et en sucre, que pour les fourrages classiques par suite d'une caramélisation très importante, la diminution enregistrée dans cet essai semble cependant anormalement élevée. Elle aurait vraisemblablement été réduite si les betteraves avaient été mises en cossettes plus petites et moins épaisses avant d'être déshydratées.

L'estimation de la valeur énergétique nette des betteraves à partir de la teneur en matière organique digestible pose un problème. Nous l'avons estimée de deux façons :

- par la formule de BREIREM (I954) en prenant I,79 calories d'énergie nette pour l'engraissement (NKF) par g de sucre, ce qui donne :

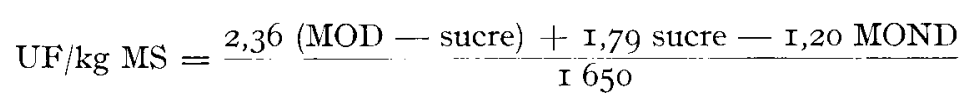

où les teneurs en matière organique digestible (MOD), en matière organique non digestible (MOND) et en sucre sont exprimées en $\mathrm{g}$ par $\mathrm{kg}$ de matière sèche.

- par la méthode employée par les auteurs allemands, belges et hollandais, en prenant 75 comme " coefficient de correction " (Wertigkeit) (KEILNER et BECKER, Ig66) :

$$
\mathrm{UF} / \mathrm{kg} \mathrm{MS}=\frac{2,36 \mathrm{MOD} \times 0,75}{\mathrm{I} 65^{\circ}}
$$

Les valeurs obtenues pour les betteraves fraîches (tabl. 2) suivant les 2 méthodes sont très voisines et comprises entre 0,85 et $0,96 \mathrm{UF}$ par $\mathrm{kg}$ de matière sèche (soit 
VALEUR ALIMENTAIRE DES BETTERAVES

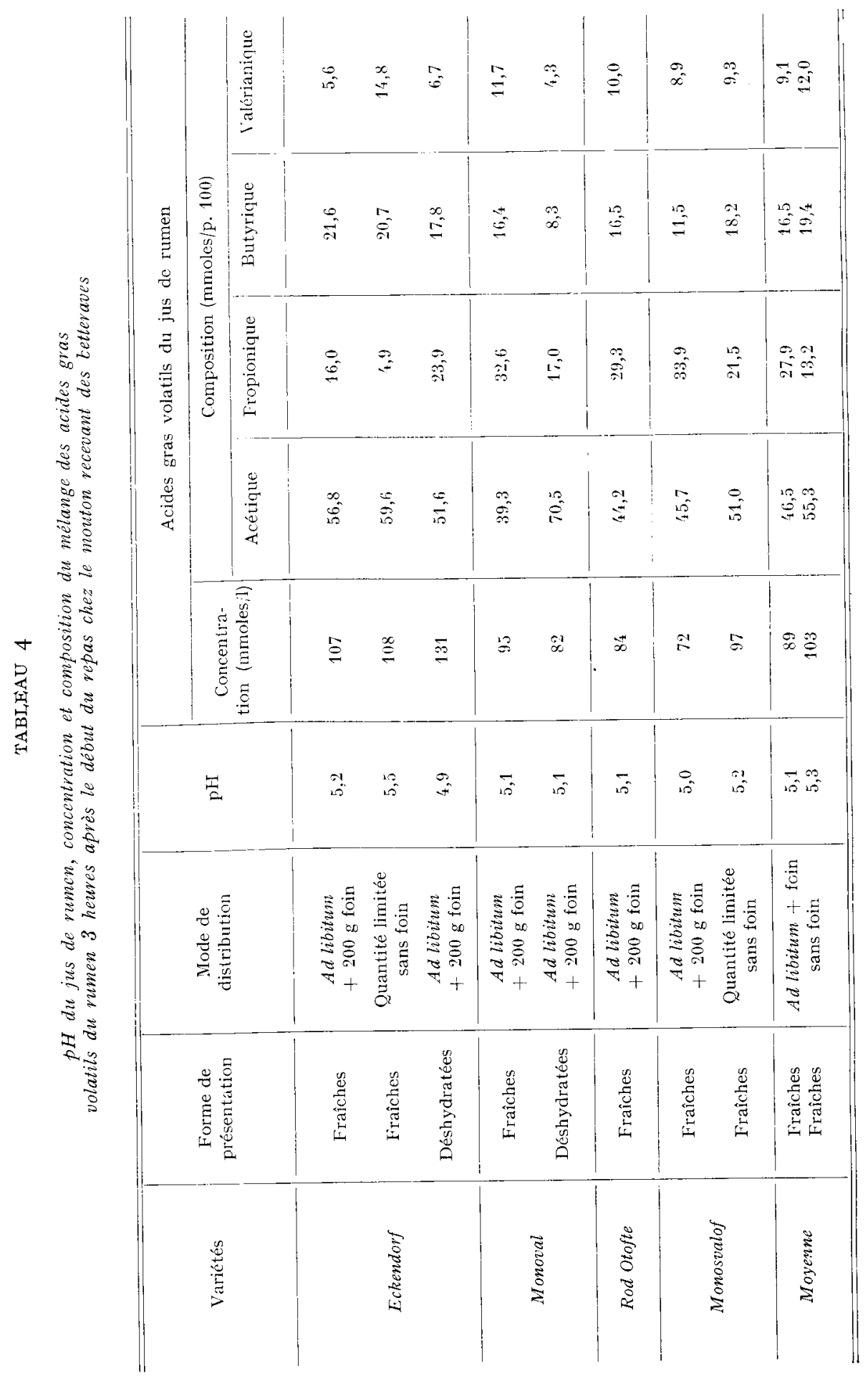


0,88 à I,oo UF $/ \mathrm{kg}$ de matière organique) suivant les variétés et suivant que les mesures sont faites avec ou sans foin. Ces valeurs correspondent très bien à celles classiquement tirées des expériences sur vaches laitières (cf. VéRITÉ, I970) o11 animaux à l'engrais. Filles doivent être cependant considérées comme des valeurs minimum, valables quand les betteraves constituent une partie importante de la ration. C'est ainsi que VAN Es et al. (I97I) ont montré que l'énergie digestible du saccharose était aussi bien utilisée par les vaches que celle de l'amidon quand le saccharose ne représentant qu'une faible fraction de la matière sèche ingérée. ( $8 \mathrm{p}$. roo de la matière sèche de l'aliment concentré, soit environ 5 p. Ioo de la matière sèche de la ration totale).

\section{Produits terminaux de la digestion}

La composition du mélange des acides gras volatils du jus de rumen a été très variable d'un échantillon de betterave à l'autre, de même qu'elle a été assez variable d'un jour à l'autre sur le même mouton ingérant une variété donnée, ce qui avait déjà été signalé par CoTTyn et BoucQuE (I970). Il se dégage cependant du tableau 4 que le jus de rumen des animaux recevant une ration à base de betteraves fraîches (soit seules, soit avec un peu de foin) a un pH très faible $(4,9$ à 5,5) et que le mélange des acides gras volatils contient une proportion élevée d'acide butyrique et surtout d'acides ayant une longueur de chaîne égale ou stupérieure à celle de l'acide valérianique (ils proviennent de la désamination des acides aminés libres de la betterave) et une proportion faible d'acide a cétique. Quant à la proportion d'acide propionique, elle est en moyenne élevée mais peut varier suivant la variété et suivant qu'il y a ou non un peu de foin dans la ration. Le type de fermentation obtenu avec ces rations à base presque exclusive de betteraves semble plus favorable à l'engraissement qu'à la production laitière.

\section{CONCLUUSION}

Cette étude montre que la digestibilité de la matière organique des betteraves est élevée (de l'ordre de 90 p. I0o), indépendante de la teneur en matière sèche de la variété (ce qui confirme les résultats trouvés par d'autres auteurs) et du niveau d'ingestion des animaux. En revanche, l'ingestibilité des betteraves pour le mouton est très variable; elle diminue quand la teneur en matière sèche de la variété augmente. Il serait intéressant de confirmer ces différences d'ingestibilité, notamment sur des bovins et d'en rechercher les causes. Enfin, la betterave peut être distribuée à volonté aux animaux sans entraîner une diminution trop importante du temps passé à ruminer à condition de maintenir un minimum de foin (environ Io p. Ioo) dans la ration. Ceci explique vraisemblablement que nous n'avons observé aucun trouble digestif chez les animaux au cours des 6 mois d'essai, malgré un pH du rumen très faible. 


\title{
SUMMARY
}

\author{
DIGESTIBILITY, NUTRITIVE, VALUE, AND VOLUNTARY \\ IN'TAKE OF BEETS WITH DIFFERENT DRY MATTER CONTENTS
}

I. The digestibility and voluntary intake of 4 varieties of beets (Rouge d'Eckendorf, Monoval, Rod-Otofte and Monosvalof), representative of the present range of dry matter contents, were measured. Studies of the 4 varicties, whose characteristics are reported in table $I$, were made on the fresh material, and in the case of 2 varieties also after dehydration. The beets were offered ad libitum and the animals also received $200 \mathrm{~g}$ of hay by day. In addition, digestibility of the fresh Eckendorf and Monosvalof varieties was measured without offering hay to the animals, but in this case beets were offered in restricted amounts. The feeding behaviour of 3 sheep out of the 6 used for the determination was recorded on graphs, and the composition of the rumen fluic, taken from sheep number 7 fitted with a fistula, was determined.

2. The dry matter content of fresh beets varied widely ( 5.5 to 28.4 P. Ioo) according to variety. The soluble carbohydrate content (59.4 to 68.2) increased at the same time is the dry matter content, but dehydration had a depressing effect. The nitrogen content was relatively variable ( $\mathrm{I} .26$ to $\mathrm{I} .92 \mathrm{p}$. I $\mathrm{IO}$ ) according to varicty and independently of the dry matter content. The same was found for the crude fiber content $: 5.8$ to $7.3 \mathrm{p}$. Ioo (table $\mathrm{r}$ ).

3. The voluntary intake of the fresh beets offered ad libitum varied considerably according to variety (tabl. 2) : 75.0 to $4 \mathbf{I} .4 \mathrm{~g}$ dry matter $/ \mathrm{kg} \mathbf{P}^{\mathbf{0 . 7 5}}$. It was even higher when the dry matter and carbohydrate content of the beets was low. These differences remained after dehydration even though the latter caused a decrease in the voluntary intake.

The fecding behaviour (table 3 ) of the experimental sheep was very diferent from that of the sheep receiving green forages. The daily time spent cating was very low $(7.3$ to Io.8 p. roo of the total time) and the beets were caten rapidly, especially those with low dry matter contents. The meals were of short-duration, but more numerous. The daily rumination time, though low (23.I to 28.2 p. Ioo of the total time when hay was added to the diet, I I.3 to I3.9 p. I oo of the total time when there was no hay), remained however definitely higher than that which would have been recorded if the same amount of dry matter had been offered as concentrate feed. This it probably the reason why we did not notice any digestive trouble in the animals during the whole trial ( 6 months).

4. The digestibility of the organic matter (table 2) of the fresh beets offered without hay ranged between 90 and $9 \mathrm{r}$ p. Ioo. The digestibility of the beets offered with some hay lay between 89,5 and $85,6 \mathrm{p}$. 100 according to variety because of the phenomenon of negative associative digestibility. The nitrogen digestibility varied widely $(72.3$ to $53.5 \mathrm{p}$. Ioo), reflecting the variation of the nitrogen content. The crude fiber digestibility was very high with little variation (86 p. 10o) when the beets given without hay.

Dəhydration brought about a large decrease in the digestibility of all the beet constituents. The decrease found seemed to be abnormally high and would probably have been reduced if the beets had been cut into smaller fragments before dehydration.

5. The rumsn fluid of the fistulated sheep fed on beets showed a low $\mathrm{pH}(4.9$ and 5.5) and a volatile fatty acid composition mainly characterized by a high butyric acid content and especially acids with a chain lenght equal to or higher than valeric acid and a low acetic acid content (table 4).

\section{RÉFÉRENCES BIBLIOGRAPHIQUES}

Brnes J. A., Davey A. W. F., r97o. Voluntary intake, digestion, rate of passage, amount of material in the alimentary tract and behaviour in cows receiving complete diets containing straw and concentrates in different proportions. Br.J. Nutr. 24, I0I3-1027.

BREIREM K., 1954. Die Netteenergie als grundlage der bewertung der futtermittel, in : NeHRing K, roo jahre Möckern. Die Bewertung der futterstoffe und undere probleme der Tiernähning. Berlin, Deutsche Akad. der Landwirtschaftswissanchaften, t. II, 97-ro8. 
Cotryn B. G,, Boucoue Ch. V., r97o. Digestibilité et valeur nutritive des betteraves fourragères riches en matière sèche. Revue de l'A griculture, 23, 489-499.

Dijkstra N. D., I953. De verteerbaarheid en vooderwaarde van bieten. Versl. Landbouwk. Onderz. $n^{r} \cdot 59-7$.

Dijkstra N. D., i960. De verteerbaarheid en voederwaarde van polyploide bieten. Versl. Landbouwk. Onderz. $n^{r}$. 66-3.

Frederiksen J., Hojland, OG A. S. Jensen. Ig64. IV Ford ø jeligheidsfors og. B. Fodersukkerroer og Klovergraesho. Forsogslaboratoriets arbog $1964: \mathrm{r} 29$.

Kellner O., Becker M., 1966. Grundzüge der Fütterningslehre (I4 Auflage). Verlag Paul Parey, Hamburg un Berlin.

Rigaud J., Journet M., I97o. Méthode de dosage des acides gras volatils dans le liquide du rumen. Ann. Biol. anim. Bioch. Biophys. 10, I 5 I-I 57.

RuckeBUSCH Y., I963. Recherches sur la régulation centrale du comportement alimentaire ché les ruminants. Thèse Doct. Sci., Université de Lyon.

Somogyr M., I952. Notes on sugar determination. J. Biol. Chem. 105, 19-23.

Van es A. J. H., Nykamp H. J., Vogt J. E., I971. The net energy content of dried sugar beet pulp and of sucrose when fed to lactating cows. Neth. J. A gric. Sci. 10, 48-56.

Vérité R., i97o. Utilisation des betteraves et des choux par les vaches laitières. Fourrages, 42, 84-88. 